\title{
Armament Research Foundation"Large complex equipment collaborative virtual maintenance training system basic technology research"
}

\author{
Yunrong Zhang, Zhili Zhang, Xiangyang Li \\ The Rocket Force University of Engineering, Xi'an 710025,China
}

\begin{abstract}
Keywords: multi person cooperative parallel ; Virtual maintenance ; concurrency control ; human-computer interaction
\end{abstract}

\begin{abstract}
At present, Large complex equipment maintenance training exists lack of physical equipment.In order to solve the problem, according to the situation of equipment and maintenance training, I built a large complex equipment multi person parallel virtual maintenance system. The system adopts the hierarchical RTI architecture based on HLA and the network communication mechanism based on DK algorithm is constructed. This ensures the consistency of the model data in Collaborative Virtual Environments. The system adopts the compound concurrency control scheme to solve the conflict problem in the multi-person cooperation. The system uses data glove and position tracker to realize human-computer interaction. The system can effectively manage the training data. In this paper, the system is implemented with the example of the maintenance and dismantling of the sealing ring of the launching equipment. The results show that the scheme can realize the virtual collaborative maintenance training of large complex equipment.
\end{abstract}

\section{INTRODUCTION}

Large complex equipment maintenance have caused problems such as training means a single, lack of equipment fault setting for less. This has seriously affected the training effect. The virtual maintenance training system can ensure the maintenance staff to experience the whole process of maintenance and help to cooperate with each other. Besides the virtual maintenance training system has low cost and can be trained repeatedly. Therefore, the research on the virtual maintenance training system of the large complex equipment has become the focus of virtual reality technology research.

At present, there are some achievements in the study of multi - person cooperative virtual maintenance of large complex equipment. In the literature [1], the system design, the overall structure, the system composition and the realization method of virtual maintenance are deeply studied. The paper uses [2] to build a general simulation system for complex equipment maintenance training. However, these two documents are designed from a single point of view, and do not involve multi user collaborative maintenance problems. In the field of concurrency control, Linebarger developed a system framework to support highly interactive team collaboration. The hierarchical concurrency control mechanism is used to deal with the collaborative activities of various types of virtual environments. Raviram et al. Proposed a concurrent control mechanism based on radial basis function neural network to deal with concurrent activities of knowledge base management system in CAD. Chen et al. Introduced the idea of token to deal with concurrent activities in an autonomous e-Assembly system. Wang Junfeng et al. Developed an integrated development platform for collaborative design in network distributed environment. They use token model to control assembly operations and avoid concurrent operations.

However, these methods are targeted or too strong, do not have a good general ability. These methods are used to solve the problem of multi person cooperation in the process of assembly step by step and do not propose a suitable communication mechanism to solve the consistency problem in distributed virtual reality. In short, most of the research in this area is to solve the problem of multi person.

The key of large scale complex equipment multi person cooperative virtual maintenance training system is the real-time interactive operation between multiple simulation systems. Due to the large number of parts and components, complex assembly model, large amount of data used in the 
network real-time communication, involving more users and the complexity of the relationship between the permissions, many key technologies need to be studied in order to realize multi-user real-time collaboration. According to the situation of equipment and maintenance training, this paper proposes a hierarchical RTI architecture based on HLA. In this paper, the communication mechanism of the system, the scheme of concurrent conflict control, the means of human-computer interaction and the methods of data collection and management are described in detail. On the basis of the above, this paper establishes a large-scale complex equipment multi person cooperative virtual maintenance development platform, which can meet the requirements of single and multi person virtual maintenance training system. Using this platform, the developer only needs to change the model, the database data, the breakdown phenomenon, the maintenance rule, the part restraint relations, the maintenance scene, the maintenance movement and so on. Readers can quickly develop a variety of large-scale complex equipment multi person collaborative virtual maintenance training system.

\section{Collaborative virtual maintenance system architecture}

\subsection{System functional requirements and task planning}

In view of the problems in the maintenance and training of large and complex equipment, it is difficult for the equipment to be dismantled, the multi person cooperative training is difficult to carry out, the personnel's safety can not be guaranteed, and the training cost is high, the functional requirements of collaborative virtual maintenance system should include the following functions. 1 Virtual maintenance model is built to realize the virtual assembly and disassembly of large complex equipment. 2 Effective access and sharing of computing resources to support collaborative virtual maintenance.3 Simulation of organization coordination in virtual maintenance of equipment.4 Real time interaction between user and non immersive virtual reality system.5 Has a friendly visual operation interface. 6 Have the data management of large-scale virtual maintenance system.7 Establish and maintain a unified virtual assembly scene.8 Users are able to get feedback by stereo display, sound output, etc.

\subsection{System architecture}

The system uses multiple computers to form a local area network to build a collaborative simulation environment. At present, there are mainly distributed cooperative and centralized coordination among multi computer. Distributed collaboration is to receive the input after the node, the first to complete the key computing, and then the results to the server or other nodes for arbitration, the failure of the arbitration is a retrospective state. Centralized coordination is that the server collects input current existing nodes, unified computing, distributed back to the node. Because of the large number of large and complex equipment parts, the design process and the users of the collaborative assembly process are more, the use of these two systems can not meet the requirements of synchronization and real-time.

HLA is an open, object-oriented, distributed interactive simulation architecture. RTI is the specific implementation of the HLA interface specification. At present, RTI has three structural models, which are centralized, distributed and hierarchical. Its hierarchical model combines the characteristics of centralized and distributed to solve this problem. The structure model has a central server for performing some global operations. A set of local servers are set up under the central server. Each local server is responsible for a group of members of the service request. This structure can reduce the delay of global operation and improve the efficiency of simulation. The sub server performs some local operations to reduce the coupling degree and improve the execution efficiency. In view of the requirement of multi person cooperative virtual maintenance for large complex equipment and the characteristics of RTI hierarchical structure model, a collaborative virtual assembly scheme based on RTI is proposed. The overall design schematic is shown in Figure 1. 


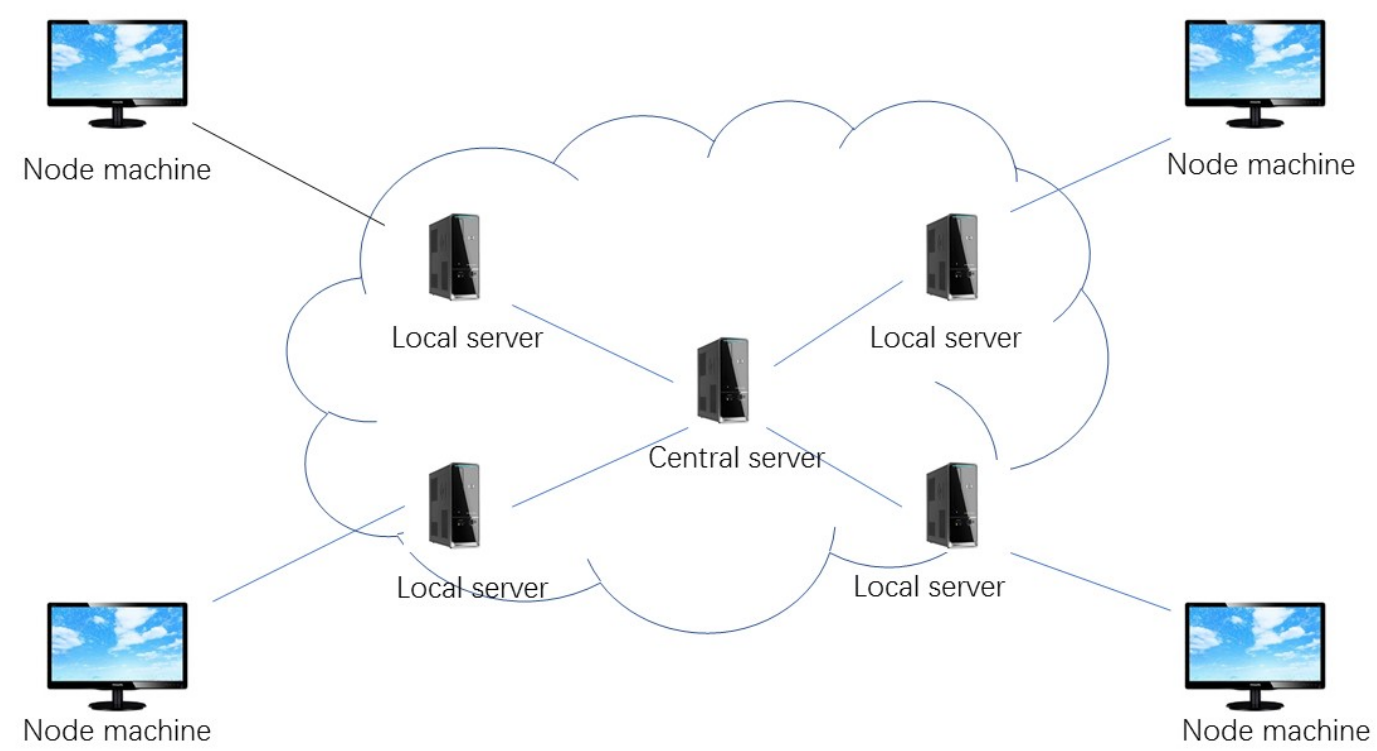

FIGURE 1.1. collaborative virtual assembly architecture

When working, the system starts the RTI service first, and then starts the central server. The central server sends a registration to the RTI to create a collaborative assembly to create a collaborative assembly environment. Each user is configured hardware parameters according to user name and IP address to the RTI application to join the Federal Assembly. The central server judges whether the user is legal. If it is legal, it is allowed to join to create the same virtual environment as the master. The connection between the user node machine and the interactive device is shown in figure 2 .

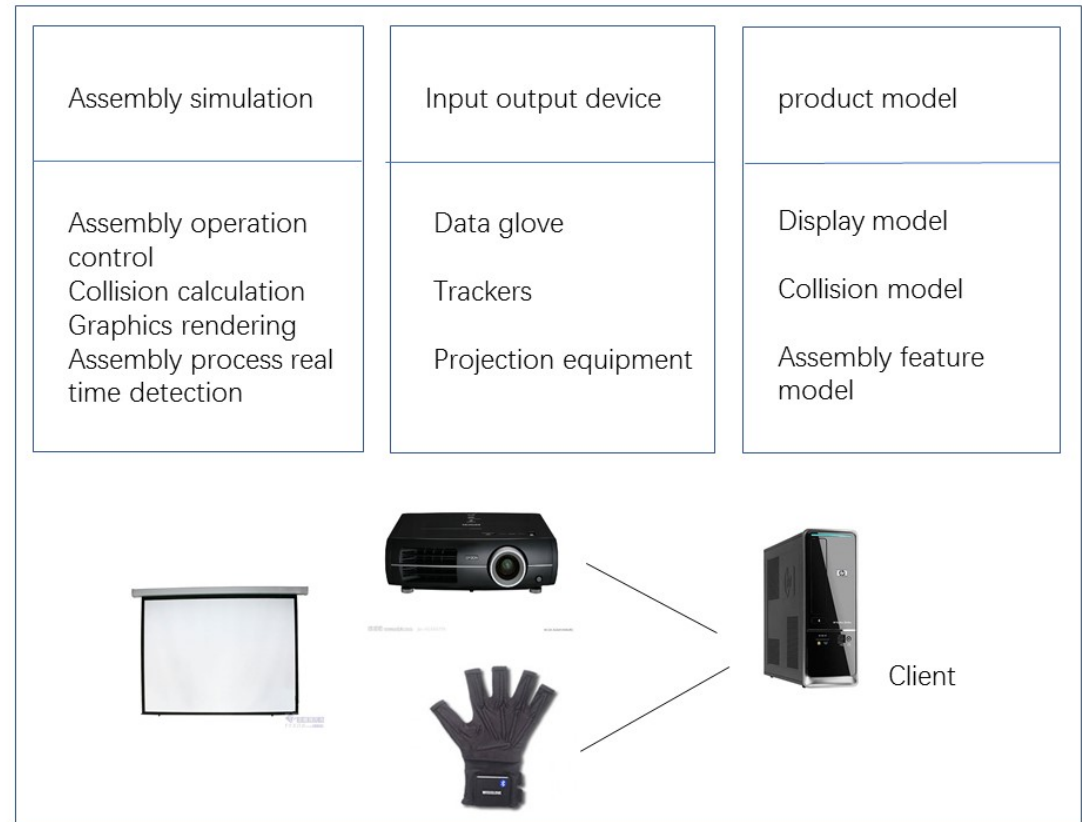

FIGURE 1.2. connection relation between user node machine and interactive device

\section{System operation platform development}

The system running platform is mainly composed of visual operation interface, network communication mechanism based on DK algorithm and the bottom database. 


\subsection{Visual operation interface}

Visual operation interface is the first interface when the user enters the virtual maintenance system. Users can apply to join the virtual maintenance activities through the interface can also grasp the progress of virtual maintenance activities through the interface. Users can also communicate and negotiate with other participants. Therefore, the visual operating interface is an important window for the feedback of the system. The visual operation interface is composed of a control button, a display window of the equipment, a language whiteboard and the current task status display bar. According to the requirements of development, we can use Photoshop, Flash, Lab VIEW and GL Studio image processing software and virtual instrument design software to develop accordingly.

3.2 Network communication mechanism based on DK algorithm

Dead reckoning algorithm is a solution to ensure the synchronization of network transmission delay. This algorithm is widely used to solve the problem of synchronization of online games. In this paper, the algorithm is applied to the research of the virtual maintenance training system for large complex equipment. The algorithm plays an important role in the communication between the server and the host server. Each user has a maintenance process entity and a DR model on its own client. The client operation process is synchronized to the DR model, and finally passed to the server through the DR model. When the client is operating in the client, the client generates a sequence of instructions that are sent to the server via the DR model. The server queues the instructions. At the same time the server will update all customer operations through the DR model feedback to each client. Through practice and optimization, the paper gives the core code of dead reckoning algorithm which is more suitable for network transmission. Through practice and optimization, the paper gives the core code of dead reckoning algorithm which is more suitable for network transmission.

$\mathrm{X}, \mathrm{Y}$, Cof: real array

$\mathrm{N}$ : int

Process $(\mathrm{X}, \mathrm{Y}, \mathrm{N}, \mathrm{cof})\{$

Define variables:

S: real array

I,J,K: int

PHI, PF, B: real number

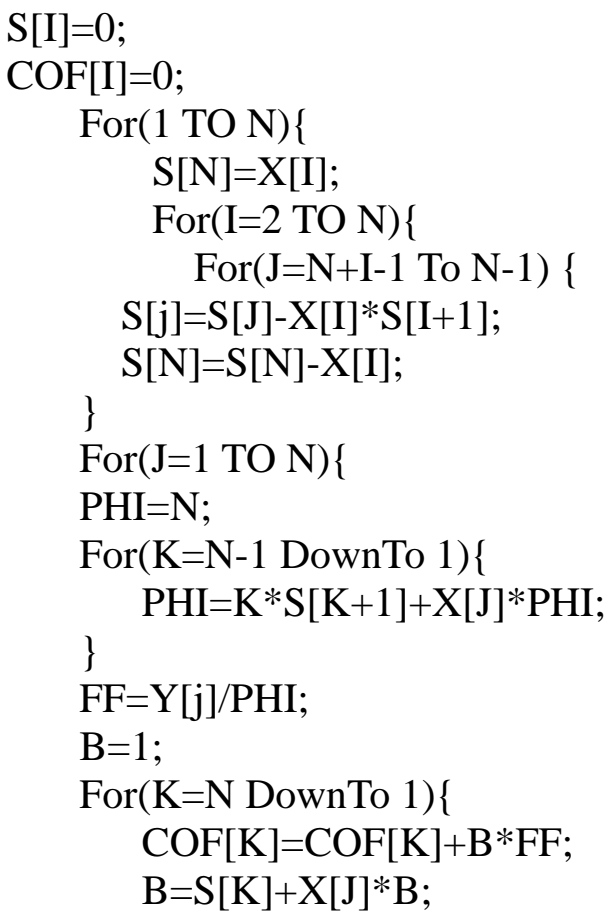




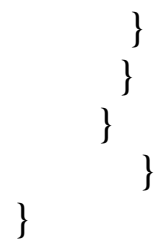

\section{Complex concurrent conflict control}

Large scale complex equipment multi person cooperative virtual maintenance training system allows multiple users to operate the same virtual shared object at the same time. There is a need to coordinate the conflict between concurrent behaviors. Large scale complex equipment multi person cooperative virtual maintenance training system must provide a concurrency control strategy to ensure the order of concurrent behavior. At this stage, some of the concurrency control methods are the token method, lock method, operation queue method, ownership management, etc. These methods have achieved the effect of concurrency control to some extent, but there are also some problems. For example, the token mechanism increases the system response time, and lock method is not easy to control the length of lock time. In this paper, based on the combination of the permission method and the operation queue method, I added a time threshold method to limit the operator's long-term occupation of the manipulated object.

4.1Multiple permissions + operation queue method

For the same objects, the operator has more than one. These operators have different operating privileges on the object. Some operators can be carried out on the object to be disassembled and assembled, and some operators can modify the object to be manipulated, and some operators can only view the manipulated object, no modification or disassembly of power. We sort these different permissions according to the numbers and assign them to different operators. When a number of operators at the same time the operation of the object to be issued for the operation of the application, the system control module will be sorted according to their permissions. The operator of the same authority is sorted according to the order of the application time. After the formation of the operation queue, if there are other operators to apply to join the operation queue, can be directly inserted into the operating queue according to the size of its operating authority.

4.2 Time threshold method and the law of compulsory use right

But there are problems with this approach. Low permissions token user might have been cut, and other related operations to continue, so that the whole system card lag phenomenon. In order to solve this problem, a time threshold method is proposed. When someone is waiting for the object to be limited to its operating time, the operation must be completed within the specified time. When the time is over, the system will be forced to take back the right to use. This method is mainly for some users in the use of the forgotten after the use of the right to return. If it is not yet completed, the participants can re apply for use. The system will assign the right of use to him. Of course, in some cases, with higher privileges of the user to be operated really long time operator and there has been a high user permissions to jump the queue, this makes the low authority users have been waiting for the state. These low authority users can't do other steps while waiting. A long time will affect the overall operation of the system and the phenomenon of hysteresis. The system shall give the user the right to use the object for more than two times. This situation is to ensure the smooth operation of the system, of course, can also be a certain degree of protection of the participants in the event of the fairness of the joint operation.

\section{Realization of virtual maintenance training system}

5.1The hardware and software foundation of virtual maintenance training system

The development of the large-scale complex equipment multi person cooperative virtual maintenance training system includes Unity3D, C\#, Pro/E and XML, and the main interactive 
equipment is data glove and position tracker. Unity3D is the development tool of virtual maintenance scene. The 3D model of the large complex equipment is created by Pro/E for the two development and data format conversion. C\# is used for the creation of the system running platform as well as the scripting tool of Unity3D. The model information and fault information in the database are created by creating a simplified mathematical model and a XML data structure. This facilitates the analysis and processing of XML data based on the.NET simulation platform, and makes the fault logic model established by the fault tree structure relatively fixed. In the system expansion, update and two development, just modify the relevant information in the XML. The man-machine interactive equipment used in large scale complex equipment multi person cooperative virtual maintenance training system is data data glove and position tracker. The data glove provides the hand gesture information for the virtual hand, which ensures the consistency between the virtual hand and the user gesture. But it is not possible to change the position and rotation of the virtual hand in three dimensions. The spatial position tracking system is mainly used to track and obtain the position and direction information of people or objects in space. Through the calculation and analysis of the corresponding velocity vector and acceleration vector, so that the virtual environment in the simulation of the object model for real-time interactive control. The position and rotation information of the hand is measured in real time with the help of position tracking device. The system transfers the information to the virtual hand program to control the movement of the virtual hand in virtual space.

5.2 Virtual maintenance training system for launching equipment of tank vehicle

The large and complex equipment, which is represented by the launching equipment of tank vehicles, is composed of many parts. Large complex equipment maintenance must cooperate with each other, and have good communication and coordination between each other. The system simulates the fault of the elastic chain, oil pipe leakage, seal ring leakage, gasket aging and so on. The system not only deepens the understanding of the structure of the equipment, but also improves the cooperation ability of the participants. Compared with the existing multi person virtual maintenance training system, this system has achieved the innovation in the network communication, cooperation, conflict control mechanism and so on. It can realize the user cooperation maintenance on the ordinary PC. The system has been put into practical application.

\section{Concluding Remarks}

Large complex equipment maintenance training is research hotspots of Distributed Virtual Reality Technology.This paper presents hierarchical RTI architecture based on HLA. The whole process and framework of system development are given In the paper. The network communication mechanism based on DK algorithm and the method of database management are studied. This paper puts forward a scheme of multiple concurrency control for "multiple rights + operation queues". The basic hardware and software of the system are expounded. The virtual maintenance training system for a certain type of launch vehicle is developed. The related technology of the system is verified. Experiment shows that the system can well support the development of all kinds of large and complex equipment.

\section{References}

[1] Jianping Hao. Theory and technology of Virtual Maintenance Simulation [M]. Beijing: National Defence Industry Press 2008:219-277.

[2] Yuhang Yang, Mandi Su, Hui Qiao. General simulation system of complex equipment maintenance training [J]. Journal of system simulation, 2008,(11):2885-2888+2892.

[3] Xiaogang $\mathrm{Xu}$, Liming $\mathrm{Ou}$, Chengyong Shao. Single / multi person virtual maintenance training platform [J]. Acta Armamentarii, 2012,(07):886-891.

[4] Xijin Zhen, Dianliang Zhen, Xiumin Fan. Research on multi user modeling and collaborative technology of distributed virtual assembly system [J]. Journal of system simulation, 2008, 
(S1): 124-131.

[5] Bin Li, Hanguo Cui, Zhengmin Li. Collaborative virtual maintenance system based on shared subnet for Petri net [J]. china shiprepair, 2015, (05): 45-49.

[6] Sihang Zhou, Zhenyu Liu, Jianrong Tan. Concurrent operation control method for collaborative maintenance disassembly simulation [J]. Journal of Zhejiang University (Engineering Science Edition), 2012, (12): 2133-2140.

[7] Xiangyang Li, Zhili Zhang, Qinhe Gao, etal. Research on Simulation and development of fault diagnosis training system for large weapon equipment $[\mathrm{J}]$. Journal of system simulation, 2009, (21): 6770-6773.

[8] Chao Zhao, Xingxin Li, Kong Xia. Concurrent operation control method for collaborative virtual maintenance [J]. Computer system application, 2014, (10): 198-201.

[9] Shiqi Li, Yaqing Feng, Junfeng Wang, etal. Collaborative virtual disassembly training platform in Network Environment [J]. Computer aided engineering, 2013, (04): 82-86.

[10]Liming $\mathrm{Ou}$, Xiaogang $\mathrm{Xu}$, Jianguo Wang, etal. Collaborative virtual maintenance and its key technologies [J]. Ship Science and Technology, 2010, (11): 122-125.

[11] Yanfang Ma. Research and design of virtual collaborative experiment [D]. Beijing University of Posts and Telecommunications, 2011. 